\title{
X-Ray Absorption and Photoelectron Spectroscopies Using Total Reflection X-Rays ${ }^{\dagger}$
}

\author{
Jun KaWaI*, Shinjiro Hayakawa**, Yoshinori KitaJima*** and Yohichi GohSHI** \\ *Department of Materials Science and Engineering, Kyoto University, \\ Sakyo, Kyoto 606-01, Japan \\ **Department of Applied Chemistry, University of Tokyo, \\ Hongo, Tokyo 113, Japan \\ *** Photon Factory, National Laboratory for High Energy Physics, \\ Oho, Tsukuba 305, Japan
}

\begin{abstract}
X-Ray absorption and X-ray photoelectron spectroscopic measurements of specular surfaces using grazing incidence soft $\mathrm{X}$-rays carried out by the present authors are summarized. The merits of using a sample current induced by totally reflected X-rays are discussed. The probing depth of the sample current has been found to be restricted to within a very shallow surface. The application of the sample current X-ray absorption experiment to the depth profile analysis of flyash powders is described. Very clear X-ray photoelectron spectra excited by totally reflected X-rays are for the first time reported.
\end{abstract}

Keywords X-Ray absorption spectroscopy, X-ray photoelectron spectroscopy, total reflection X-ray

Total reflection (TR) or grazing incidence X-ray analysis $\left(\mathrm{GIXA}^{1}\right)$ make it possible to perform: (1) surface sensitive analysis, (2) trace analysis, and (3) surface layer structure analysis. The GIXA technique has so far been used with X-ray fluorescence (GIXF) ${ }^{2}$ and X-ray diffraction (GIXD) or scattering (GIXS). ${ }^{1}$ However, GIXA has a potential to be combined with X-ray absorption spectroscopy (XAS) and X-ray photoelectron spectroscopy (XPS). We overview in the present paper our ongoing work concerning total reflection X-ray absorption spectroscopy (TRXAS) and total reflection X-ray photoelectron spectroscopy (TRXPS) using soft $\mathrm{X}$-rays. Soft X-rays ranging from $1500-2500 \mathrm{eV}$ have an advantage in that the total reflection experiment is easy because the critical angle of the total X-ray reflection is larger (a few degrees) than that of hard X-rays.

\section{Total Reflection X-Ray Absorption Spectroscopy}

X-Ray absorption spectroscopy in the soft X-ray region has been technically difficult because of problems concerning (i) the lack of appropriate $X$-ray sources and (ii) a too large $X$-ray absorption coefficient for preparing sufficiently thin samples. The first problem was solved by the development of synchrotron radiation facilities.

+ Presented at the 5th Workshop on Total Reflection X-Ray Fluorescence Spectroscopy and Related Spectroscopical Methods, October 17-19, 1994, Tsukuba, Japan.
An answer to the second problem was found in the 1980s using various techniques for measuring the quantum yields, which are equivalent to the $\mathrm{X}$-ray transmission method. ${ }^{3}$ Thus, measuring the X-ray absorption spectra in the soft $\mathrm{X}$-ray region is now not difficult. The quantum yield techniques are appropriate for applying the total X-ray reflection technique to X-ray absorption spectroscopy.

Figure $1^{4}$ illustrates various methods for measuring the X-ray absorption spectra. Figure la shows the traditional X-ray transmission method, by which one can obtain the X-ray absorption $(\mu)$ by calculating $\mu=\log \left(I_{0} /\right.$ $I$ ), where $I_{0}$ is the incident $\mathrm{X}$-ray intensity, and $I$ is the transmitted X-ray intensity. Since $\mu$ becomes very large in the soft X-ray region, it is practically difficult to prepare sufficiently thin films to transmit soft X-rays. Therefore, the method illustrated in Fig. 1a is technically impossible. We sometimes receive a sample which cannot form a film, and sometimes must analyze samples without any pretreatment because some kind of pretreatment might change the chemical state of the sample. The traditional X-ray transmission method of XAS is thus inappropriate to be combined with the total reflection technique.

The methods illustrated in Figs. $1 b, c$ and $d$ are variations of the electron yield method. If the $\mathrm{X}$-rays are strongly absorbed by matter, the photoelectrons, Auger electrons, or secondary electrons are strongly emitted. We can use an electron energy analyzer prior to detection in order to select these electrons with respect 
(a)

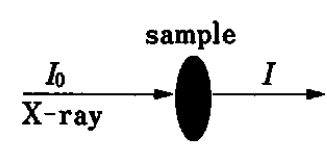

(c)

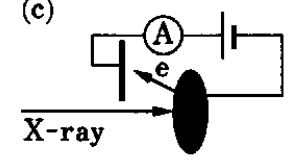

(e)

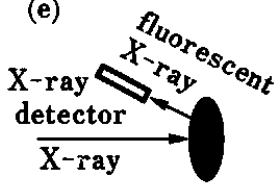

Fig. 1 Various methods for measuring the X-ray absorption spectra. (a) Transmission method, (b-d) electron-yield method, and (e) fluorescence-yield method (taken from ref. 4).

to their origins. The sample current (Fig. 1d) contains information concerning all of the electrons emitted from the sample surface. Although the sample current detection method is simple, it is the most surface sensitive, as described below. These electron-yield methods are easily combined with total X-ray reflection technique.

Figure le illustrates the X-ray fluorescent yield method used to measure the X-ray absorption. This method can also be combined with the total X-ray reflection technique. The X-ray fluorescent yield is also surface sensitive when used with total X-ray reflection. The detection limit of the fluorescent-yield method is usually lower than that of the sample current method.

Figure $2^{5}$ illustrates the experimental setup for measuring the total reflection X-ray absorption spectra using the method described in Figs. $1 \mathrm{~b}$ and $\mathrm{d}$, which was performed on beam-line BL-11B at the Photon Factory, National Laboratory for High Energy Physics (KEK), Tsukuba, Japan. Monochromatized synchrotron radiation impinged on a semiconductor single crystal wafer in an ultra-high vacuum; also, the sample current and high energy electron (kinetic energy was larger than a few hundreds electron volts) intensity emitted from the sample surface were measured while changing the glancing angle. We measured a GaAs wafer; the resulting glancing-angle dependence of these two signals are shown in Fig. $3 a .^{6}$ Figure $3 b^{6}$ shows the numerically simulated glancing-angle dependence of signals emitted from 0 (surface), 10 and $100 \AA$ depths from a GaAs surface. Comparing Figs. $3 a$ and $b$, it can be seen that the experimental sample current curve in Fig. 3a is intermediate between simulated 0 and $10 \AA$ curves in Fig. 3b; also, the experimental curve for electrons having kinetic energy larger than $500 \mathrm{eV}$ (Fig. 3a) is intermediate between the simulated 10 and $100 \AA$ curves (Fig. 3b). It can therefore be concluded that the sample current probes a $0-10 \AA$ surface, and high energy $(>500 \mathrm{eV})$ electrons probe $10-100 \AA$ depth from the surface. Note here that the experimental result given in Fig. 3a contains a geometrical factor, $1 / \cos \theta$. The sample current contains all the electrons, the major portion of which is secondary electrons whose kinetic energy is less than $50 \mathrm{eV} .^{7}$ These low energy electrons are emitted from the top of the surface. Thus, the probing depth of the sample current is shallower than that of those electrons having a kinetic energy greater than $500 \mathrm{eV}$.

We have measured the X-ray absorption spectra of chemisorbed sulfur on a GaAs surface using the $S \mathrm{~K}_{\alpha} \mathrm{X}$ ray fluorescence yield and the sample current. X-Ray fluorescence was detected by a proportional counter. Although the X-ray fluorescence yield exibited a clear S K absorption edge, the sample current did not show any edge structure around the $\mathrm{S} K$ threshold region. Thus, the detection limit of the X-ray fluorescence method is lower than that of the sample current method. This was because although the sample current contained the total signal from the minor component (S) and major components ( $\mathrm{Ga}$ and $\mathrm{As}$ ), the X-ray fluorescence yield only contained the sulfur signal.

The surface of a single crystal $\mathrm{Si}$ wafer is usually oxidized, having a thickness of $c a .5 \AA$. We measured the Si K X-ray absorption spectra of a 2-in. Si wafer using a grazing incidence $X$-ray beam. We observed the difference between the surface and bulk chemical states while changing the glancing angle of the incident $X$-rays during the measurement of the X-ray absorption spectra. Figures $4 a$ and $b^{6}$ show the Si $\mathrm{K}$ edge absorption spectra measured by a glancing angle larger (Fig. 4a) and smaller (Fig. 4b) than the critical angle of the X-ray total reflection. Figure $4 \mathrm{~b}$ is the total X-ray reflection X-ray absorption spectra. Peaks at 1842 and $1849 \mathrm{eV}$ are characteristics of $\mathrm{Si}$ and $\mathrm{SiO}_{2}$, respectively. The spectrum measured by the sample current under the total reflection condition is the most surface sensitive because the relative oxide peak intensity is strongest, as shown in Fig. 4b; the spectra which are measured by a sample current or electrons $\left(E_{\mathrm{k}}>1200 \mathrm{eV}\right)$ at angles larger than the critical angle contain bulk information. The sample current spectra are somewhat saturated, i.e. the peak intensity is too weak, due to the reason described by Stöhr. ${ }^{8}$

Sample-current detection XAS is very surface sensitive when it is combined with the total reflection X-ray technique. However, sample current detection alone is also surface sensitive. To illustrate the surface sensitiveness of sample-current detection, we measured sulfur X-ray absorption spectra of a coal fly-ash powder by using both the sample current and the X-ray fluorescence yield (Fig. $5^{9}$ ). In this case the incident Xrays were not totally reflected, because the analyte was in the powder form on a flat sample holder. The sample current method is used to detect the surface (ca. $100 \AA$ ) oxide layer of $\mu \mathrm{m}$ size particle powders when the $X$-rays impinge on the sample from the normal direction. The sample current XAS of fly ash shown in Fig. 5b has a 


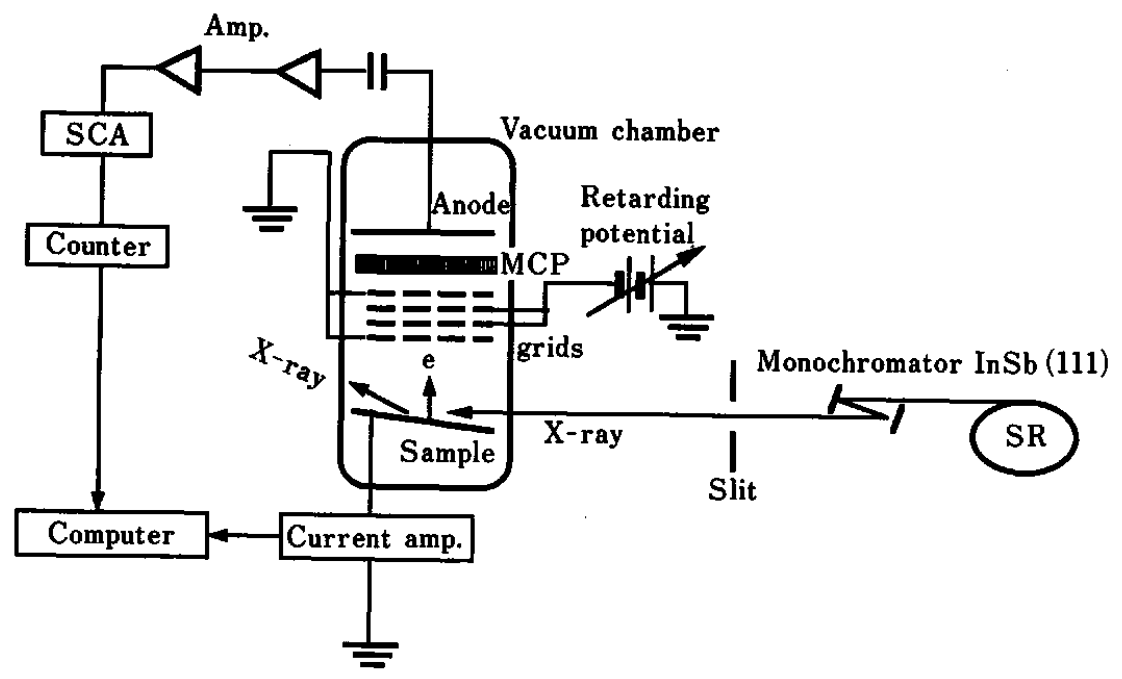

Fig. 2 Schematic illustration of the experimental setup used to measure the total reflection X-ray absorption spectra of semiconductor wafers (taken from ref. 5).
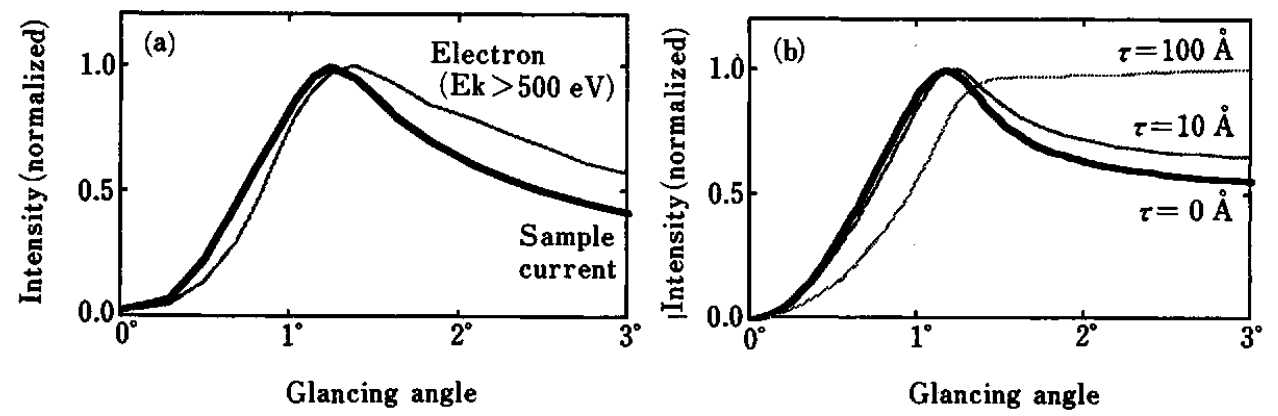

Fig. 3 (a) Experimental glancing-angle dependence of the electron intensity, whose kinetic energy is larger than $500 \mathrm{eV}$ (thin solid line), and the sample current including all electrons emitted from the sample (heavy solid line) for a $2 \mathrm{keV} \mathrm{X-ray}$ beam impinging on a GaAs wafer. (b) Calculated glancing-angle dependence of the electron intensity emitted from places shallower than 0,10 and $100 \AA$ from the surface (taken from ref. 6).
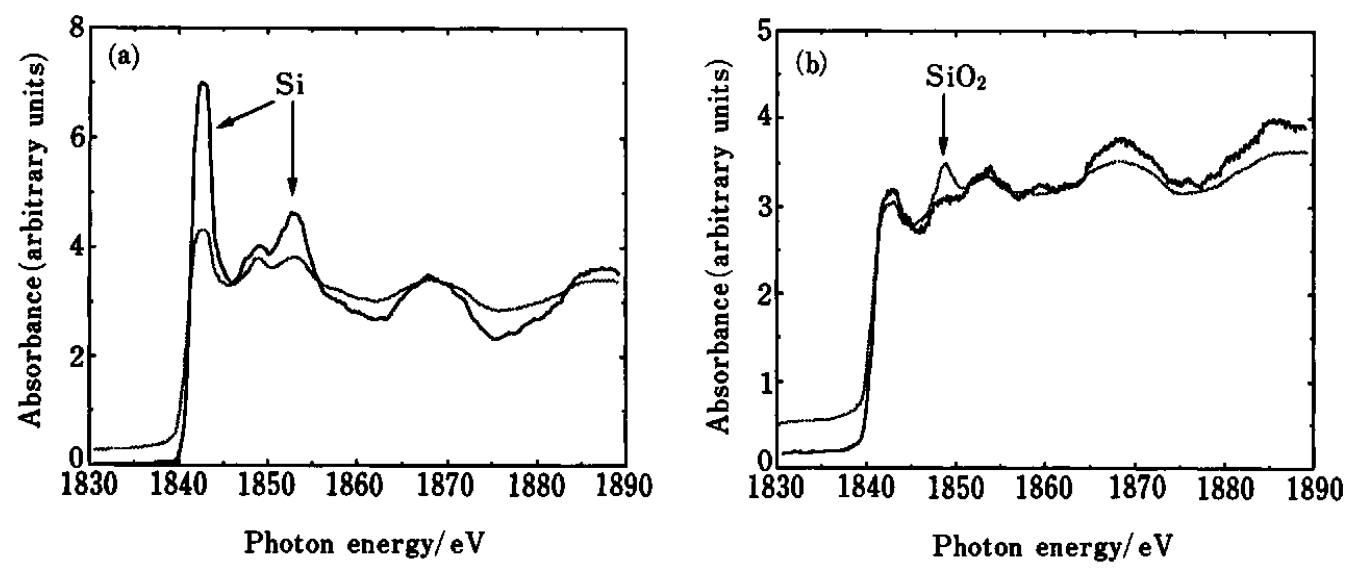

Fig. 4 (a) Measured X-ray absorption spectra of a Si wafer using the sample current (dotted line) and the partial electron yield (solid line). The glancing angle is larger than the critical angle of the total reflection. (b) Measured X-ray absorption spectra of a $\mathrm{Si}$ wafer using the sample current (dotted line) and the partial electron yield (solid line). The glancing angle is less than the critical angle of the total reflection (taken from ref. 6). 

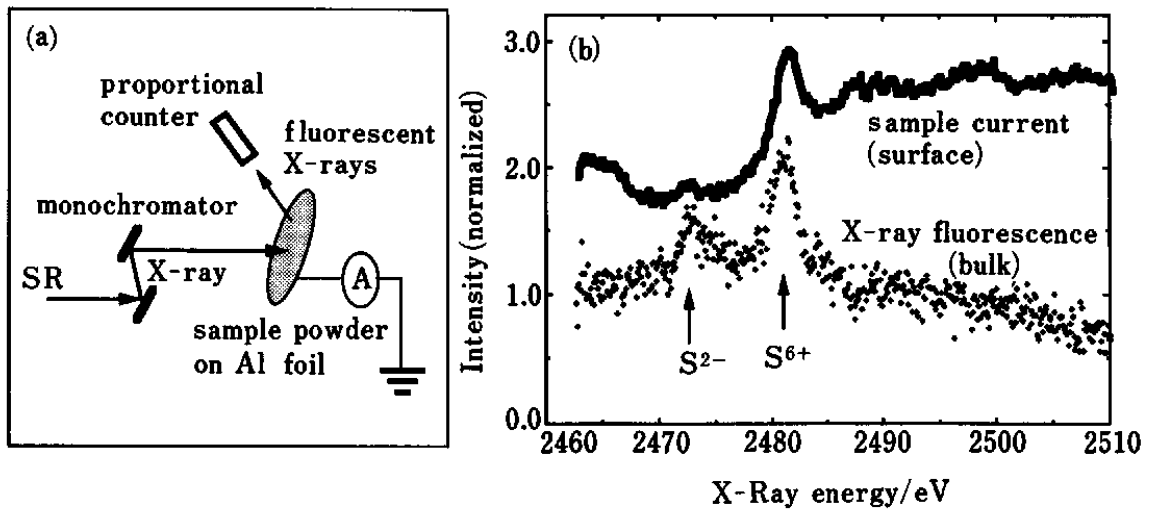

Fig. 5 (a) Experimental setup for measuring the depth selective analysis of powder samples. (b) Measured sulfur K X-ray absorption spectra of coal fly ash (taken from ref. 9).

strong $\mathrm{S}^{6+}$ peak, which originated from the surface oxide layer; the X-ray fluorescent yield XAS shows both $\mathrm{S}^{2-}$ and $\mathrm{S}^{6+}$ peaks. The $\mathrm{S}^{2-}$ existed in a deep place of a flyash particle, which had not been oxidized in a combustion process of coal. We can obtain information concerning the surface and bulk chemical states simultaneously by measuring the sample current and the $\mathrm{X}$-ray fluorescent yield concurrently.

We can not define the critical angle of the X-ray total reflection when the incident $\mathrm{X}$-ray energy ranges over $1000 \mathrm{eV}$. To measure EXAFS (extended X-ray absorption fine structure) we need to measure at least $500 \mathrm{eV}$ above the absorption edge. On the other hand, NEXAFS (near edge X-ray absorption fine structure) spectroscopy measures $50 \mathrm{eV}$ above and below the absorption edge. However, in this X-ray region, the absorption coefficient drastically changed pathing through the X-ray edge. Thus, the critical angle as well as the X-ray evanescent depth drasticaly changed both above and below the threshold energy. The saturation effect described above and this probing depth change are two problems in interpreting the total reflection X-ray absorption spectra.

Total reflection X-ray absorption spectral measurements have been reported by other researchers for the $\mathrm{Si}_{3} \mathrm{~N}_{4} / \mathrm{GaAs}$ system $^{10}$, a $\mathrm{Si} / \mathrm{Ge} / \mathrm{Si}$ monolayer system ${ }^{11}$ and an aqueous solution surface. ${ }^{12}$ Total reflection XAS has been reviewed by Stöhr ${ }^{8}$ and Pandya et al. ${ }^{13}$

\section{Total Reflection X-Ray Photoelectron Spectros- copy}

If we measure the photoelectron energy distribution using the same system as shown in Fig. 2, but attached to a high resolution electron energy analyzer, we can measure the total reflection X-ray excited photoelectron spectra (TRXPS). Henke ${ }^{14}$ first demonstrated the total reflection effect regarding the photoelectron intensity; recently, Jach and coworkers ${ }^{15-18}$ demonstrated the use

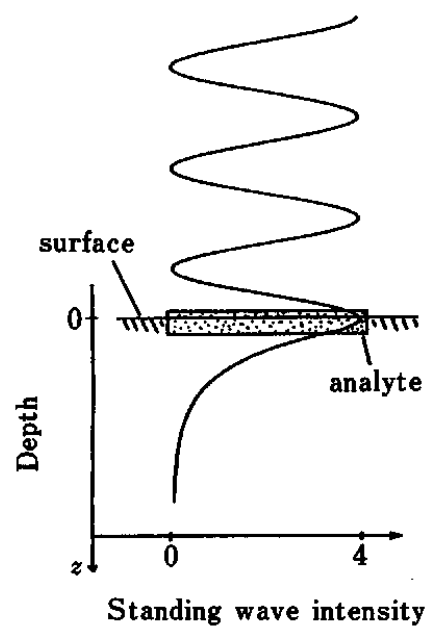

Fig. 6 Schematic illustration of the surface sensitivity of total reflection X-ray photoelectron spectroscopy (taken from ref. 19).

of total reflection X-ray photoelectron spectroscopy for surface chemical state analysis. In this method the incident X-ray energy is fixed, and the electron kinetic energy detected is scanned. Using this method, it has been predicted that the surface sensitivity is enhanced, as shown in Fig. 6. ${ }^{19}$ The standing wave at the surface is four times as strong as the incident $\mathrm{X}$-ray intensity. Contrary to this, the total reflection $\mathrm{X}$-ray fluorescence intensity is twice because the incoming and outgoing Xrays exite the analyte at the surface.

It is also predicted that the total reflection X-ray photoelectron spectroscopy has a characteristic that the spectral backgrounds originating from inelastically scattered electrons are reduced to less than one half (Fig. $7 \mathrm{a}^{19}$ ). This is because the excitation X-rays exist on and near the surface region, and that the excited electrons are restricted in this region. Thus, photoelectrons hardly lose their kinetic energy through 

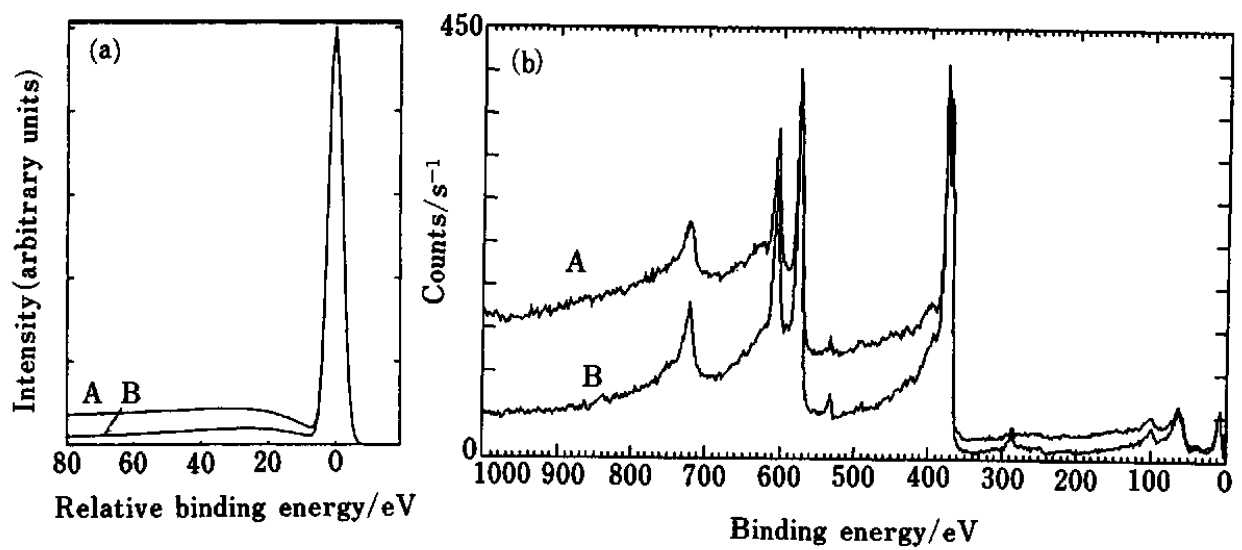

Fig. 7 (a) Numerical simulation of a comparison between (A) normal incidence and (B) total reflection XPS (taken from ref. 19). (b) Measured X-ray photoelectron spectra changing the glancing angle of the incident X-rays: (A) above the critical angle and (B) below the critical angle.

inelastic scattering. On the other hand, normal incidence XPS excites electrons located deeper than $1000 \AA$ in a solid; these electrons lose their kinetic energy while traveling from a deeper place to the surface of the solid. These energy loss electrons form very high intensity backgrounds in the XPS spectra. We have measured the total reflection XPS of Ag evaporated on a Si wafer (Fig. 7b). It is found from this figure that the background of the TRXPS is proven to be reduced, as predicted in ref. 19. A comparison between Tougaard's background formula ${ }^{20}$ and the experimental TRXPS makes it possible to determine the inelastic mean free path of a particular kinetic energy electron in a solid.

It is shown that the total reflection X-ray technique, which is used with the sample current measurement, enhances the surface sensitivity. The probing depth of this method is typically $5 \AA$. The direct electron detection method excited by the total reflection X-rays probes somewhat deeper places ( $10 \AA$ ) compared with the sample current method. This is because electron detectors sometimes detect higher kinetic energy electrons more efficiently. The sample current is also surface sensitive, even when used with normal incidence $\mathrm{X}$-rays $(20-100 \AA)$. We have shown a clear total reflection X-ray photoelectron spectrum for the first time. It has been shown that the photoelectron spectrum of Ag excited by total reflection X-rays has low backgrounds due to the reduction of inelastic electron scattering. This method has a potential for determining the electron mean free path in solids experimentally.

This is a summary of going work financially supported by a Grant-in-Aid for Scientific Research (No. 05555226) from the Ministry of Education, Science and Culture, Japan, and experiments have been performed under the approval of the Photon Factory Program Advisory Committee (PF-PAC No. 90-234, 92G189, 94G179 and 94G365).

\section{References}

1. D. K. G. de Boer, Phys. Rev., B44, 498 (1991).

2. Y. Yoneda and T. Horiuchi, Rev. Sci. Instrum., 42, 1069 (1971).

3. J. Stöhr, "NEXAFS Spectroscopy", Springer Series in Surface Science 25, pp. $114-161$, Springer-Verlag, Berlin, Heidelberg, 1992.

4. J. Kawai, H. Adachi, S. Hayakawa, S. Zheng, K. Kobayashi, Y. Gohshi, K. Maeda and Y. Kitajima, Spectrochim. Acta, 49B, 739 (1994).

5. J. Kawai, S. Hayakawa, Y. Kitajima, Y. Yakata, T. Urai, K. Maeda, M. Fujinami, Y. Hashiguchi and Y. Gohshi, Appl. Phys. Lett., 63, 269, 3238 (1994).

6. J. Kawai, S. Hayakawa, Y. Kitajima, S. Suzuki, K. Maeda, T. Urai, H. Adachi, M. Takami and Y. Gohshi, Proc. Japan Acad., 69B, 179 (1993).

7. D. Briggs and M. P. Seah, "Practical Surface Analysis by Auger and X-ray Photoelectron Spectroscopy", Wiley, Chister, 1983.

8. J. Stöhr, "NEXAFS Spectroscopy", Springer Series in Surface Science 25, p. 126, Springer-Verlag, Berlin, Heidelberg, 1992.

9. J. Kawai, S. Hayakawa, F. Esaka, S. Zheng, Y. Kitajima, K. Maeda, H. Adachi, Y. Gohshi and K. Furuya, Anal. Chem., 67, 1526 (1995).

10. D. Jiang, N. Alberding, A. J. Seary and E. D. Crozier, J. de Phys., C8, 861 (1986).

11. G. Tourillion, E. Dartyge, A. Fontaine, M. Lemonnier and F. Bartol, Phys. Lett., A121, 251 (1987).

12. H. Tanida, I. Watanabe and H. Watarai, Book of Abstracts, 8th Intern. Conf. on X-Ray Absorption Fine Structure, Berlin, 1994.

13. K. I. Pandya and D. C. Koningsberger, "Analysis of Microelectronic Materials and Devices", ed. M. Grasserbauer and H. W. Werner, pp. 637-655, Wiley, Chister, 1991.

14. B. L. Henke, Phys. Rev., A6, 94 (1972).

15. M. J. Chester, T. Jach and S. Thurgate, J. Vac. Sci. Technol., B11, 1609 (1993). 
16. M. J. Chester and T. Jach, Phys. Rev., B48, 17262 (1993).

17. T. Jach, M. J. Chester and S. Thurgate, Nucl. Instrum. Methods Phys. Res., A347, 507 (1994).

18. T. Jach, M. J. Chester and S. Thurgate, Rev. Sci. Instrum., 65, 339 (1994).

19. J. Kawai, M. Takami, M. Fujinami, Y. Hashiguchi, S.
Hayakawa and Y. Gohshi, Spectrochim. Acta, 47B, 983 (1992).

20. S. Tougaard, Surf. Sci., 216, 343 (1989).

(Received March 4, 1995)

(Accepted April 17, 1995) 